\title{
How to Optimize Images for Oral Presentations \\ Tim Miller
}

There is perhaps no more common tool used in the communication of materials research findings than the data graphic. Ranging from simple $x-y$ plots to the hugely complex three-dimensional visualizations used in the biological sciences, data graphics have the capacity to convey volumes of numerical and spatial information in an incredibly concise format. The use of figures is nearly ubiquitous throughout the sciences, and we see them appear in articles, posters, and live presentations. Yet while researchers go to great lengths to obtain and interpret the information contained within these images, too few spend the time to carefully consider how that information is displayed. In order for data to have an impact on a viewer, it must not only be scientifically accurate, it must also be presented in a manner the viewer can comprehend, and which is appropriate to the medium in which it will be viewed. More often than not, the figures we see in live presentations are precisely the same figures that appeared (or will appear) in publication. In this article, I offer some simple tips on designing figures specifically for projected display during talks and lectures. The figures presented here are also available in powerpoint on the MRS Bulletin Web site (www.mrs. org/bulletinMar10) to view on screen.

\section{Consider the Color Scheme}

Perhaps the most obvious difference between figures designed for printing and those designed for projection is the color of the background (see Figure 1). We generally draw figures for publication with black (or colored) data, titles, and axes over a white background. This is appropriate for printing, because on paper, empty space is white (if you turn the printer off, you get a white page). Yet in projection, empty space is black (if you turn the projector off, you get a black screen). Thus the clearest projected image is one where the information is drawn in white (or in color) and the background is left black. This scheme is not only less tiring on the eye of the viewer, it also allows the speaker to move physically inside the projected area, without casting a shadow of his body on the screen or blinding himself with the light from the projector.

\section{Avoid the Small Multiple}

Another common practice in figures designed for printing is the use of the socalled "small multiple," a series of several

Figure 1

Figure 2

Figure 3
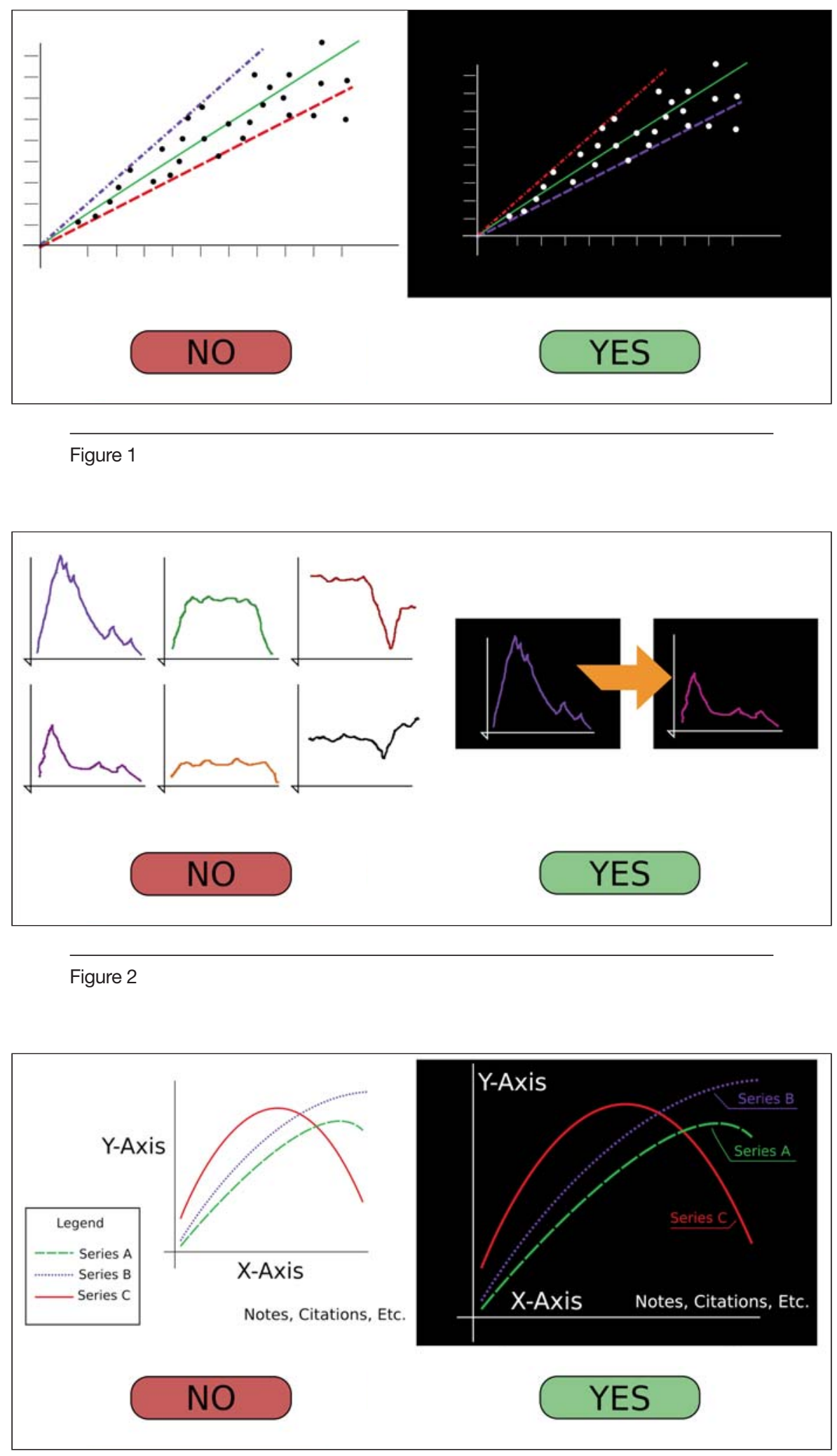
figures in an array (see Figure 2). This technique is extremely useful for published figures, as it encourages clear comparison between a large number of different data series. Yet it is the high resolution of the printed page, combined with the fact that the reader has an unlimited amount of time to cross-reference one figure to the next that makes these comparisons possible. When images are projected, the resolution is far lower, and the viewer can only consume the information for as long as the speaker chooses to display it. Thus, small multiple figures are often difficult to make out, and lose much of their utility. In cases where you want your audience to compare two (or more) data series, it is better to enlarge each of them to fill the entire projected area, and then cycle through them one at a time, returning if necessary to the original image (Slide A - Slide B - Slide C - Slide A), in order to reinforce the comparison.

\section{Fill the Space}

One technique that is common to figures designed for projection or printing is to make the data itself as large as possible. This is especially important when designing figures for public display, since projected images will vary widely in actual size, depending on the size and orientation of the projector, and in apparent size, depending on how far away the audience member is from the screen. A simple way to maximize the available space is to move the labels, titles, and legends inside the axes (see Figure 3). It is rare that a figure will have data completely covering the plotted area, and by moving the text inside some of the unused space, you can maximize the scale. It is also clearer to have labels which directly identify the series of data, rather than a legend which associates a certain line pattern or color to a series. This allows the viewer to instantly identify the series, rather than referencing back and forth between the data and the legend.

\section{Own Every Pixel}

Perhaps the best advice related to the design of high-quality data graphics is to make sure that you are the one designing them. The software packages that are most commonly used for numerical computation or statistical analysis, and even the software interfaces that control the instruments used for data collection, usually have some built-in functionality to create data graphics. Yet the kinds of graphics these packages produce are rarely optimized for projection, and can be extremely cumbersome to manipulate after they are generated. The most effective way to exercise complete pixel-bypixel control over your images is to work with a program that is dedicated to graphic design, like Adobe Illustrator, CorelDraw, or (the slightly less-polished, but open-source, and free) Inkscape. The flexibility of a drawing program means that you can easily alter colors, move or

INTERFACES seeks articles featuring the people and profession of materials. Send proposals to Bulletin@mrs.org. edit labels and titles, and easily create multiple versions of the same figures, optimizing some for printing, and others for projection. Most drawing programs are also able to directly import numerical information in a variety of formats, ensuring the integrity of the data. While the time and (in the case of proprietary programs) cost of investing in a graphic design program may seem daunting, the quality, clarity, and impact of the images these programs can produce more than justifies the time and money spent.

While it may seem arduous to go about creating figures specifically for public presentations, it is important to remember that the communication of scientific results is not an addendum to scientific inquiry, but part of the scientific process itself. Materials researchers spend vast quantities of time and effort generating new scientific knowledge, but too often shortchange themselves when it comes to sharing that knowledge. By applying the same diligence and fastidiousness to the dissemination of your work as you do to its creation, you can both clarify the import of your results, and amplify the impact of your findings. Public talks and presentations can be a uniquely powerful and effective method to share your work, and their success can have a major impact on your career. By taking the time to create visuals for your talks that are clear, concise, and aesthetically appealing, you increase your chances for success.

Tim Miller is an independent science communications consultant. He can be reached at tim@spokenscience.com.

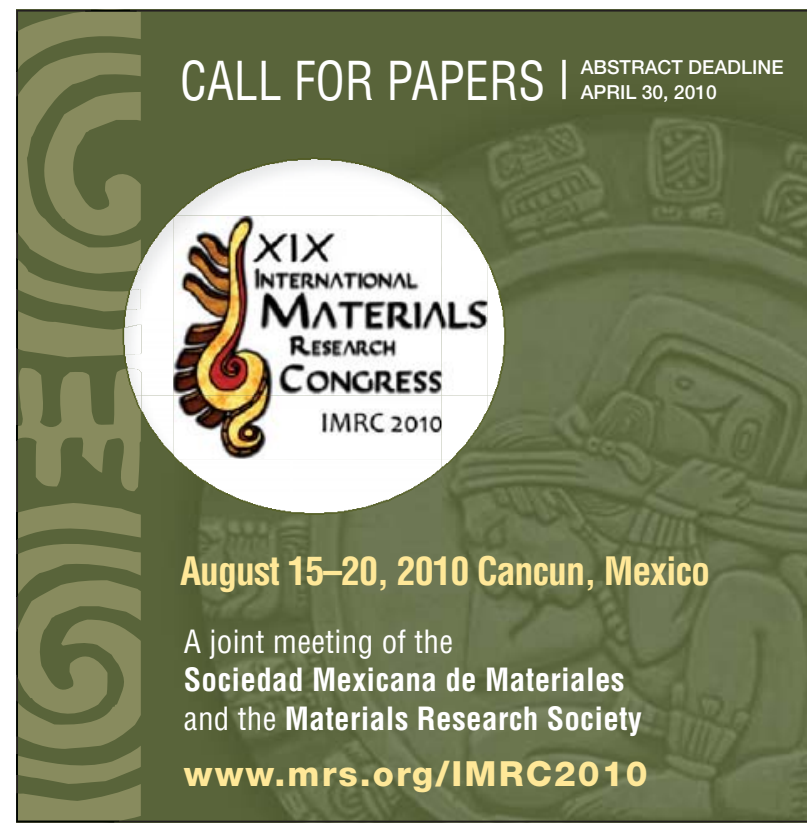

SYMPOSIA

1. Nanostructured Materials and Nanotechnology

2. Theory and Computer Simulation of Materials

3. Structural and Chemical Characterization of Metals Alloys and Compounds

4. Advanced Structural Materials

5. New Trends in Polymer Chemistry and Characterization

6. Biomaterials

7. Ecomaterials and Climate Change

8. Composite and Hybrid Materials

9. Advances in Semiconducting Materials

10. Strategies for Academy-Industry Relationships

11. New Catalytic Materials

12. NACE: Corrosion and Metallurgy

13. In Situ Microscopy and Spectroscopy of Large Surfaces and Interfaces ${ }^{\star}$

14. Photon Absorption for Solar Energy Harvesting*

15. Photovoltaics, Solar Energy Materials, and Technologies*

16. Renewable Energy and Sustainable Development

17. Domain Engineering in Ferroic Systems*

18. Ferroelectric and Piezoelectric Materials, Devices, and Applications*

19. Solid-State Chemistry of Inorganic Materials*

20. Materials and Devices for Flexible Electronics*

21. Nanomaterials for Biomedical Applications*

22. Graphene*

23. Nanostructure Applications in Cross-over Scientific and Technology Fields* 24. Advancing Materials through Scattering and Spectroscopy*

${ }^{*}$ Co-organized by MRS and MRS-Mexico 